\title{
REGULADOR VEGETAL E BIOESTIMULANTE NA INDUÇÃO FLORAL DO MARACUJAZEIRO-AMARELO EM CONDIÇÕES DE ENTRESSAFRA ${ }^{1}$
}

\author{
ELMA MACHADO ATAÍDE 2 , CARLOS RUGGIERO ${ }^{3}$, JOÃO DOMINGOS RODRIGUES ${ }^{4}$ \\ JOÃO CARLOS DE OLIVEIRA ${ }^{3}$, TERESINHA DE JESUS DÉLEO RODRIGUES ${ }^{5}$, JOSÉ RAFAEL DA SILVA ${ }^{6}$
}

\begin{abstract}
RESUMO - O objetivo do trabalho foi avaliar o efeito de regulador vegetal e de bioestimulante na indução floral do maracujazeiro-amarelo em condições não-indutivas, em Araguari-MG. Foram identificados e podados 12 ramos terciários por parcela (02-04-05), sendo 6 deles expostos de um lado da espaldeira, com luminosidade predominante pela manhã e 6 do outro lado da espaldeira, com luminosidade à tarde. $\mathrm{O}$ delineamento experimental foi em parcelas subdivididas, com 7 tratamentos principais (parcelas): $0 \mathrm{mg} \mathrm{L}^{-1}$ (testemunha); 100mg L-1; 200mg L${ }^{1}$ e $300 \mathrm{mg} \mathrm{L}^{-1}$ de regulador vegetal $\mathrm{GA}_{3}$ (i.a.); $2,08 \mathrm{~mL} \mathrm{~L}^{-1} ; 4,17 \mathrm{~mL} \mathrm{~L}^{-1}$ e 6,25 $\mathrm{mL} \mathrm{L}^{-1}$ de bioestimulante Stimulate ${ }^{\circledR}$ (i.a.), em duas aplicações foliares (09-04-02 e 09-05-02), acrescidas de espalhante adesivo Silwett ${ }^{\circledR}$ a 0,05\%. Além desses, foram utilizados 2 tratamentos secundários (subparcelas): exposição dos ramos à luminosidade da manhã e da tarde, com 4 repetições de 3 plantas por parcela. Cada subparcela foi um dos dois lados da espaldeira. As médias foram comparadas pelo teste de Tukey, a 5\% de probabilidade. Realizou-se, aos 75 dias, a avaliação nos dois lados da espaldeira do comprimento dos ramos e entrenós, número de nós, de folhas e de botões florais. As variáveis estudadas não foram influenciadas pelo uso de $\mathrm{GA}_{3}$ e Stimulate ${ }^{\circledR}$, no entanto houve diferença quando os ramos ficaram expostos à luminosidade da manhã em relação àqueles com luminosidade à tarde.
\end{abstract}

Termos para Indexação: florescimento, giberelina, Passiflora edulis Sims f. flavicarpa, stimulate

\section{PLANT REGULATOR AND BIOSTIMULANT IN YELLOW PASSION-FRUIT FLORAL INDUCTION UNDER NON INDUCTIVE CONDITION}

\begin{abstract}
The objective of the present work was to evaluate the effect of plant regulator and biostimulant in the floral induction of yellow passion-fruit under non inductive conditions in Araguari-MG. Twelve tertiary branches were identified and trimmed per plot (04/02/04), whereas six were exposed, on one side of the supporting stick, with predominant luminosity during the morning and six on the other side of the supporting stick, with luminosity during the evening. The experimental design was subdivided plots, with main treatments (plots): 0mg $\mathrm{L}^{-1}$ (control); $100 \mathrm{mg} \mathrm{L}^{-1} ; 200 \mathrm{mg} \mathrm{L}^{-1}$ and $300 \mathrm{mg} \mathrm{L}^{-1}$ of plant regulator $\mathrm{GA}_{3}$ (i.a.); $2.08 \mathrm{~mL} \mathrm{~L}^{-1} ; 4,17 \mathrm{~mL} \mathrm{~L}^{-1}$ and $6.25 \mathrm{~mL} \mathrm{~L}^{-1}$ of Stimulate ${ }^{\mathrm{TM}}$ biostimutant (i.a.), in two leaf applications (04/09/02 e 05/09/02), supplemented with Silwett ${ }^{\mathrm{TM}}$ adhesive spread at 0.05\%. Besides those treatments, secondary treatments were used (subplots): exposition of the branches to morning and evening light, with four repetitions and three plants per plot. Each subplot was one of the sides of the supporting stick. The data was submitted to the analysis of variance. In both sides of the supporting stick, the length of the branches and internodes, the numbers of nodes, of leaves and of floral buds were evaluated at 75 days after the first application of the treatments. The variables studied were not influenced by the use of $\mathrm{GA}_{3}$ and Stimulate ${ }^{\mathrm{TM}}$, however, there was difference when the branches were exposed to morning light in relationship to those exposed to evening light.
\end{abstract}

Indexation terms: flowering, gibberelin, Passiflora edulis Sims f. flavicarpa, Stimulate

\section{INTRODUÇÃO}

O maracujazeiro-amarelo (Passiflora edulis Sims f. flavicarpa Degener) é cultivado em diferentes condições climáticas, apresentando diferentes ciclos produtivos de uma região para outra. Nos últimos anos, essa cultura passou a ser vista como uma alternativa frutícola com boas perspectivas econômicas para diversos países. O Brasil destaca-se como maior produtor de maracujá, com produção de 478 mil toneladas em área de 34 mil hectares. O Estado de Minas Gerais situa-se em sexto lugar, com produção de 34 mil toneladas em dois hectares cultivados, quando comparado com as principais regiões produtoras do País, como a Bahia, Espírito Santo, São Paulo, Rio de Janeiro e Sergipe (Agrianual, 2005).

Apesar da expansão do cultivo do maracujazeiro nas diversas regiões produtoras do País, sua produção tem sido limitada nos meses de setembro a novembro (entressafra), relacionada a fatores climáticos, como o comprimento do dia, a temperatura, a radiação solar e a precipitação insuficiente (Menzel \& Simpson, 1994).

Dos fatores climáticos, o fotoperíodo desempenha importante função no florescimento do maracujazeiro-amarelo, já que esta espécie só floresce quando submetida a 12 ou mais horas de luz (Watson \& Bowers, 1965), fato esse observado nas várias regiões produtoras de maracujá do Brasil. Na região Sudeste, as baixas temperaturas e o comprimento do dia inferior a 11 horas, no outono e inverno, têm limitado a produção na entressafra (Ruggiero et al., 1996). No Sul do País, a influência do fotoperíodo é mais acentuada, enquanto, no Nordeste, precisamente na Bahia, nas condições do semi-árido, onde não havendo limitação por água, o florescimento é contínuo durante o ano, com produção na entressafra, ocorrendo o mesmo na região Norte (Ataíde et al., 2003). Como conseqüência, observam-se variações significativas nos preços do maracujá em diferentes épocas do ano.

Os melhores preços para o maracujá nas diversas regiões produtoras ocorrem nos meses de setembro a novembro, uma vez que a concentração da produção se situa nos meses de dezembro a agosto (Ruggiero et al., 1996). Denota-se, pois, a importância de os produtores estarem atentos aos aspectos relacionados ao mercado.

Estudos que promovam ajustes na fase reprodutiva do maracujazeiro, principalmente na região Sudeste, onde há limitação

\footnotetext{
${ }^{1}$ (Trabalho 196-2005). Recebido: 01-12-2005. Aceito para publicação: 25-08-2006. Parte da tese da primeira autora, para obtenção do título de Doutora em Agronomia - Produção Vegetal na FCAV/UNESP. Apóio financeiro: Maguari e Fruteza.

${ }^{2}$ D.Sc. Profa. da Faculdade de Ciências Agrárias da Universidade Vale do Rio Doce. Rua Israel Pinheiro, 2000, Bairro Universitário, CEP 35020-220, Governador Valadares - MG. E-mail: elmaataide@yahoo.com.br

${ }^{3}$ D.Sc.Prof. Titular do Departamento de Produção Vegetal da FCAV-UNESP. Via de acesso Prof. Paulo Donato Castellane, s/n, CEP 14884-900, Jaboticabal-

SP.E-mail: ruggiero@fcav.unesp.br

${ }^{4}$ D.Sc. Prof. Titular do Departamento de Botânica, Instituto de Biociências-UNESP-Botucatu. E-mail: mingo@ibb.unesp.br

${ }^{3}$ D.Sc. Prof. Titular do Departamento de Produção Vegetal da FCAV-UNESP. E-mail: jcoliveira@fcav.unesp.br

${ }^{5}$ D.Sc. Profa. Titular do Departamento de Biologia da FCAV-UNESP. E-mail: tedelro@ fcav.unesp.br

${ }^{6}$ M.Sc. Viveiros Flora Brasil - Plantas e Assessoria. Araguari-MG. E-mail: j.rafael.silva@uol.com.br
} 
fotoperiódica ao florescimento, tornam-se importantes. Uma das alternativas com vista a contornar essa limitação, pode ser a técnica da indução floral com emprego de reguladores vegetais. Dentre os principais grupos, com possibilidade de utilização, estão as auxinas, giberelinas, citocininas, etileno, retardadores e inibidores (Taiz \& Zeiger, 2004), além de bioestimulantes (Casillas et al., 1986).

Dentre os reguladores vegetais utilizados no controle da floração de muitas espécies frutíferas, as giberelinas são utilizadas para induzir a floração em espécies sensíveis ao fotoperíodo e/ou ao frio (Rêgo, 1984). Enfatizam que a resposta à indução, nestas espécies, geralmente está associada ao aumento do nível de giberelinas endógenas. Em trabalhos com laranjeiras lima Sorocaba, com aplicações de $\mathrm{GA}_{3}$, Pereira (1997) verificou redução da florada. Com laranjeiras Pêra, Castro et al. (1998) observaram incremento da produção de frutos com bioestimulante Stimulate ${ }^{\circledR}$. Enquanto Sanches (2001), pesquisando limeiras ácidas com uso de $\mathrm{GA}_{3}$, constatou aumento da produção de frutos na entressafra. Já com laranjeiras Pêra, Santos et al. (2003) observaram aumento do número de inflorescências com bioestimulante e inibição da formação de flores tardias e redução do número de frutos com $\mathrm{GA}_{3}$. Verifica-se que, apesar de esta técnica ser uma ferramenta bastante efetiva no manejo da floração de muitas espécies, muitas vezes os resultados são contraditórios. Isso se deve em parte a fatores referentes à aplicação, absorção e ao estádio fenológico da planta (Goldschmidt et al., 1998). Para Silva et al. (2003), em cada espécie vegetal, os hormônios podem exercer distintas funções no controle do desenvolvimento e função dos órgãos florais.

Neste contexto, não foram encontrados relatos na literatura com emprego de reguladores vegetais e bioestimulantes visando à indução floral em maracujazeiro. Deste modo, o objetivo deste trabalho foi avaliar o efeito de regulador vegetal e de bioestimulante na indução floral de maracujazeiro-amarelo em condições nãoindutivas, em Araguari-MG

\section{MATERIAL E MÉTODOS}

O experimento foi realizado em um pomar de maracujazeiroamarelo seleção Maguari, em Araguari-MG, no período de abril a setembro de 2002. As plantas estavam com um ano e dois meses de idade, conduzidas em espaldeiras com um fio de arame a 1,80 m do solo, espaçamento de $3 \times 5 \mathrm{~m}$, orientadas aproximadamente na posição norte e sul, promovendo diferença de luminosidade entre os dois lados da planta. O clima da região é tropical quente e úmido, seco no inverno, com área experimental localizada a $18^{\circ} 46^{\prime} 82^{\prime \prime}$ de latitude sul e $48^{\circ} 00^{\prime} 60^{\prime \prime}$ de longitude de W, com altitude de $946 \mathrm{~m}$, medidos com GPS. As adubações foram as convencionais, adotadas para o maracujazeiro-amarelo na região, com 6 adubações de cobertura por planta, sendo 3 com $230 \mathrm{~g}$ de $14-00-41$ e 3 com $160 \mathrm{~g}$ de cloreto de potássio e $160 \mathrm{~g}$ de sulfato de amônia. Utilizou-se a irrigação por gotejamento, observando o balanço hídrico da região.

O delineamento experimental foi em parcelas subdivididas, com 7 tratamentos principais (parcelas): $0 \mathrm{mg} \mathrm{L}^{-1}$ (testemunha); $100 \mathrm{mg}$ $\mathrm{L}^{-1} ; 200 \mathrm{mg} \mathrm{L}^{-1}$ e $300 \mathrm{mg} \mathrm{L}^{-1}$ de GA (i.a.); e 2,08mL L $\mathrm{mL}^{-1} ; 4,17 \mathrm{mLL}^{-1}$; e T, $-6,25 \mathrm{~mL} \mathrm{~L}^{-1}$ de bioestimulante Stimulate ${ }^{\circledR}$ (i.a.). As soluções foram aplicadas em duas pulverizações foliares (09-04-02 e 09-05-02), ao entardecer, acrescidas de espalhante adesivo Silwet ${ }^{\circledR}$ a $0,05 \%$, utilizando-se de pulverizador costal manual, com bico cônico. Foram gastos $500 \mathrm{~mL}$ da solução por planta. Além desses, foram utilizados 2 tratamentos secundários (subparcelas): exposição dos ramos à luminosidade, predominante pela manhã ou à tarde, com 4 repetições. Cada parcela foi constituída por 5 plantas, com 3 plantas úteis. Cada subparcela foi um dos dois lados da espaldeira. O produto comercial de $\mathrm{GA}_{3}$ usado foi Fitogib e do bioestimulante foi o Stimulate ${ }^{\circledR}$, que contém em sua fórmula $0,009 \%$ de cinetina (citocinina), $0,005 \%$ de ácido giberélico (giberelina) e 0,005\% de ácido indolbutírico (auxina).
O produto comercial do espalhante adesivo utilizado foi Silwett ${ }^{\circledR}$. Foram identificados 12 ramos terciários por parcela, sendo 6 ramos expostos de um lado da espaldeira, com luminosidade pela manhã, e 6 ramos do outro lado da espaldeira, com luminosidade à tarde. Os ramos foram podados (02-04-02), deixando-se 7 nós por ramo, visando a uniformizar seus comprimentos. Aos 15 dias após a poda, conduziu-se apenas uma brotação por ramo, eliminando as demais brotações. Essas brotações que permaneceram, foram o objeto de estudos das variáveis estudadas. Até então, as avaliações feitas foram prévias, buscando-se uniformidade do material experimental.

Após 75 dias da aplicação dos tratamentos, foram avaliados o comprimento dos ramos e entrenós, número de nós, de folhas e de botões florais. A análise estatística foi feita considerando os tratamentos com regulador vegetal e bioestimulante nas parcelas e, nas subparcelas, a exposição dos ramos às duas condições de luminosidade. As médias das variáveis foram comparadas pelo teste de Tukey, a 5\% de probabilidade.

\section{RESULTADOS E DISCUSSÃO}

Aos 75 dias após a primeira aplicação dos tratamentos, conforme apresentado na Tabela 1 , verificou-se que o comprimento dos ramos e entrenós, número de nós, de folhas e de botões florais não diferiram significativamente entre os tratamentos.

As aplicações com $\mathrm{GA}_{3}$ e Stimulate foram feitas buscandose um balanço hormonal que favorecesse o maior número de botões florais em maracujazeiro, em condições não-indutivas, ou seja, com fotoperíodo inferior a 12 horas/luz, o que, na região em estudo, foi de abril a agosto (Figura 1). Entretanto, as diferenças observadas não foram significativas. Talvez as concentrações utilizadas não tenham sido suficientes para induzir a floração. Essa observação encontra respaldo em Castro \& Melotto (1989), que relataram que a absorção é necessária para que os reguladores vegetais possam exercer sua atividade. As respostas em floração às aplicações de reguladores vegetais, segundo reportam Etienne et al. (1993), são muito variáveis, devendo, em parte, aos fatores concernentes à aplicação, absorção, transporte e metabolismo do produto e como citado por Goldschmidt et al. (1998).

Verifica-se que, além dos aspectos comentados, resposta à floração com uso de reguladores vegetais, tanto pode ser de indução como de inibição. Como observado por Almaquer et al. (1993) e Pereira (1997), Sanches (2001) e Santos et al. (2003), em trabalhos desenvolvidos com citros com emprego de $\mathrm{GA}_{3}$, assim como, Castro et al. (1998) e Santos et al. (2003) utilizando Stimulate ${ }^{\circledR}$.

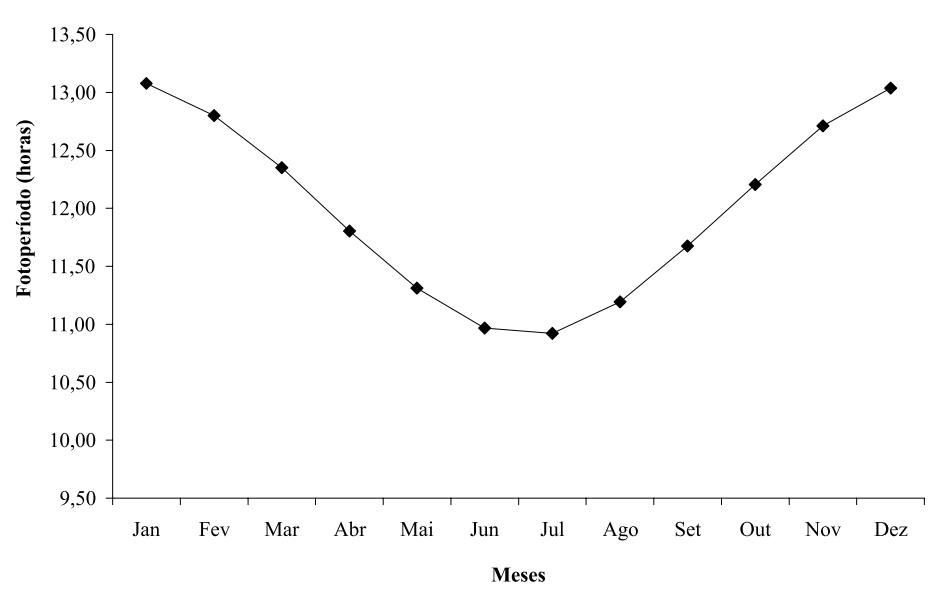

FIGURA 1 - Fotoperíodos médios mensais, referentes ao período de janeiro a dezembro de 2002 para região de AraguariMG. Instituto de Geociêcias Aplicadas da Universidade Federal de Uberlândia-MG 
TABELA 1 - Comprimento dos ramos e entrenós, número de nós, de folhas e de botões florais de maracujazeiro-amarelo, em função da aplicação de regulador vegetal e bioestimulante e da exposição dos ramos à luminosidade, aos 75 dias após a aplicação dos tratamentos. Araguari-MG.

\begin{tabular}{|c|c|c|c|c|c|}
\hline Tratamentos & $\begin{array}{l}\text { Comprimento } \\
\text { dos ramos }(\mathrm{cm})\end{array}$ & $\begin{array}{l}\text { Comprimento } \\
\text { dos entrenós } \\
(\mathrm{cm})\end{array}$ & $\begin{array}{l}\text { Número } \\
\text { de nós }\end{array}$ & $\begin{array}{l}\text { Número } \\
\text { de folhas }\end{array}$ & $\begin{array}{c}\text { Número } \\
\text { de botões } \\
\text { florais/ } \\
\text { ramo }\end{array}$ \\
\hline Testemunha & $17,6 \mathrm{a}$ & $1,5 \mathrm{a}$ & $9,5 \mathrm{a}$ & $9,5 \mathrm{a}$ & $1,9 \mathrm{a}$ \\
\hline $\mathrm{GA}_{3} 100 \mathrm{mg} \mathrm{L}^{-1}$ & $24,8 \mathrm{a}$ & $1,7 \mathrm{a}$ & $11,0 \mathrm{a}$ & $11,0 \mathrm{a}$ & $2,6 \mathrm{a}$ \\
\hline $\mathrm{GA}_{3} 200 \mathrm{mg} \mathrm{L}^{-1}$ & $24,9 \mathrm{a}$ & $1,5 \mathrm{a}$ & $11,6 \mathrm{a}$ & $11,5 \mathrm{a}$ & $2,4 \mathrm{a}$ \\
\hline $\mathrm{GA}_{3} 300 \mathrm{mg} \mathrm{L}^{-1}$ & $22,3 \mathrm{a}$ & $1,8 \mathrm{a}$ & $10,1 \mathrm{a}$ & $10,0 \mathrm{a}$ & $2,6 \mathrm{a}$ \\
\hline Stimulate $2,08 \mathrm{~mL} \mathrm{~L}^{-1}$ & $17,4 \mathrm{a}$ & $1,3 \mathrm{a}$ & $9,3 \mathrm{a}$ & $9,3 \mathrm{a}$ & $2,6 \mathrm{a}$ \\
\hline Stimulate $4,17 \mathrm{~mL} \mathrm{~L}^{-1}$ & $24,4 \mathrm{a}$ & $1,7 \mathrm{a}$ & $11,3 \mathrm{a}$ & $11,2 \mathrm{a}$ & $2,7 \mathrm{a}$ \\
\hline Stimulate $6,25 \mathrm{~mL} \mathrm{~L}^{-1}$ & $17,2 \mathrm{a}$ & $1,4 \mathrm{a}$ & $9,3 \mathrm{a}$ & $9,2 \mathrm{a}$ & $2,3 \mathrm{a}$ \\
\hline Teste $\mathrm{F}$ & $0,63^{\mathrm{NS}}$ & $0,50^{\mathrm{NS}}$ & $0,53^{\mathrm{NS}}$ & $0,57^{\mathrm{NS}}$ & $0,20^{\mathrm{NS}}$ \\
\hline DMS $(5 \%)$ & 21,96 & 1,12 & 6,23 & 6,11 & 2,86 \\
\hline Exposição dos ramos & $\begin{array}{l}\text { Comprimento } \\
\text { dos ramos }(\mathrm{cm})\end{array}$ & $\begin{array}{l}\text { Comprimento } \\
\text { dos entrenós } \\
(\mathrm{cm})\end{array}$ & $\begin{array}{l}\text { Número de } \\
\text { nós }\end{array}$ & $\begin{array}{l}\text { Número } \\
\text { de folhas }\end{array}$ & $\begin{array}{l}\text { Número } \\
\text { de botões } \\
\text { florais }\end{array}$ \\
\hline Luminosidade manhã & $31,1 \mathrm{~A}$ & $1,9 \mathrm{~A}$ & $13,8 \mathrm{~A}$ & $13,8 \mathrm{~A}$ & $3,3 \mathrm{~A}$ \\
\hline Luminosidade Tarde & $11,3 \mathrm{~B}$ & $1,2 \mathrm{~B}$ & $6,8 \mathrm{~B}$ & $6,7 \mathrm{~B}$ & $1,6 \mathrm{~B}$ \\
\hline Teste $\mathrm{F}$ & $62,58 * *$ & $65,66 * *$ & $85,94 * *$ & $85,38 * *$ & $39,42 * *$ \\
\hline DMS (5\%) & 5,21 & 0,20 & 1,59 & 1,59 & 0,57 \\
\hline Interação T x E & $1,82^{\mathrm{NS}}$ & $1,99^{\mathrm{NS}}$ & $1,62^{\mathrm{NS}}$ & $1,65^{\mathrm{NS}}$ & $1,90^{\mathrm{NS}}$ \\
\hline C.V. (\%) Parcelas & 62,71 & 43,78 & 36,68 & 36,18 & 70,57 \\
\hline C.V. (\%) Subparcelas & 44,22 & 23,45 & 27,78 & 28,01 & 41,91 \\
\hline
\end{tabular}

Quanto à exposição dos ramos à luminosidade, entre os dois lados da espaldeira, observou-se diferença para as variáveis estudadas, nas quais maiores médias foram obtidas em condições de luminosidade pela manhã (Tabela 1). Esse comportamento das plantas em resposta à luminosidade pode ser esclarecido em relatos de Vasconcellos \& Duarte Filho (2000), onde os mesmos reportam que, no sistema de condução em espaldeira, forma-se um emaranhado de ramos sobrepostos, nos quais as folhas mais internas recebem pouca luz e apresentam taxa fotossintética muito baixa. Isso leva, em algumas situações, as folhas a funcionarem como drenos. A interação entre os tratamentos com regulador vegetal e bioestimulante e a exposição dos ramos à luminosidade não foi significativa para os parâmetros estudados (Tabela 1).

Ainda é limitado o conhecimento sobre os vários mecanismos que afetam a fisiologia da planta, principalmente os de ordem hormonal. Portanto, é importante desenvolver novos estudos, com emprego de reguladores vegetais e bioestimulantes em maracujazeiro-amarelo, visando a obter o controle da floração, assim como a antecipação de produção para épocas oportunas de mercado.

\section{CONCLUSÕES}

1) O regulador vegetal e o bioestimulante não proporcionaram aumento do número de botões florais de maracujazeiro-amarelo.

2) As condições de luminosidade pela manhã favoreceram o maior comprimento dos ramos e entrenós, número de nós e de botões florais.

\section{REFERÊNCIAS}

AGRIANUAL 2005: anuário da agricultura brasileira. São Paulo: FNP, Consultoria \& AgroInformativos, 2004. p. 352-358.
ALMAQUER, V.; ESPINOZA, J. R.; CAMPBELL, R. J. Forced production in citrus trees with the application of growth regulators in México. In: ANNUAL MEETING OF THE INTERAMERICAN SOCIETY FOR TROPICAL HORTICUlTURE, 3., 1993, Santo Domingo. Proceedings... p.105-112.

ATAÍDE, E. M.; RUGGIERO, C.; SILVA, J. R. Uso de reguladores vegetais. In: SIMPÓSIO BRASILEIRO SOBREACULTURADO MARACUJAZEIRO, 6., 2003, Goytacazes. Anais... Goytacazes: UENF, 2003. 1 CD-ROM.

CASILLAS, V. J. C.; LONDOÑO, I. J.; GUERRERO, A. H.; BUITRAGO, G. L. Analisis cuantitativo de la aplicacion de cuatro bioestimulantes en el cultivo del rábano (Raphanus sativus L.). Acta Agronomica, Palmira, v.36, n.2, p.185-195, 1986.

CASTRO, P. R. C.; MELLOTO, E. Bioestimulantes e hormônios aplicados via foliar. In: BOARETO, A. E.; ROSOLEM, C. A. Adubação foliar. Campinas: Fundação Cargill, 1989. v.1, cap. 8, p. 191-235.

CASTRO, P. R. C.; PACHECO, A. C.; MEDINA, C. L. Efeitos de stimulate e de micro-citros no desenvolvimento vegetativo e na produtividade da laranja pêra (Citrus sinensis L. Osbeck). Scientia Agrícola, Piracicaba, v.55, n.2, p.338-341, 1998.

ETIENNE, H.; SOTTA, B.; MONTORO, P.; MIGINIAC, E.; CARRON, M. P. Relations between exogenous growth regulators and endogenous índole-3 acetic acid and abscisic acid in the expression of somatic embriogenesis in Hevea brasiliensis (Mull. Arg.). Plant Science, Lucknow, v.88, p.91-96, 1993.

GOLDSCHMIDT, E. E.; TAMIM, M.; GOREN, R. Gibberellins and flowering in citrus and other fruit trees: Acritical analysis. Acta Horticulture, Wagenenigen, n.463, p.201-216, 1998.

MENZEL, C.M.; SIMPSON, D.R. Passionfruit. In: SCHAFFER, B.; ANDERSEN, P.C. (Ed.). Handbook of environmental physiology of fruit crops. Boca Raton: CRC Press, 1994. v. 2, p. 225-241. 
PEREIRA, I.A.M. Época da indução e evocação floral em Cítrus spp e efeito do $\mathrm{GA}_{3}$ em seu florescimento. 1997, 81f. Tese (Doutorado em Agronomia) - Universidade Federal de Lavras, Lavras, 1997.

RÊGO, G.M. Micropropagação de plantas através da cultura de tecidos. Cruz das Almas: EMBRAPA, 1984. 17 p. Apostila do II Curso Intensivo Nacional de Fruticultura.

RUGGIERO, C.; SÃO JOSÉ, A. R.; VOLPE, C. A.; OLIVEIRA, J. C.; DURIGAN, J. F.; BAUMGARTNER, J. G.; SILVA, J. R.; NAKAMURA, K.; FERREIRA, M. E.; KAVATI, R.; PEREIRA, V. Maracujá para exportação: aspectos técnicos da produção. Brasília: EMBRAPA, 1996. 64p. (FRUPEX).

SANCHES, F.R. Efeito de ácido giberélico na floração da lima ácida 'Tahiti' (Citrus latifólia Tan.). 2001, 78f. Dissertação (Mestrado em Agronomia)-Faculdade de Ciências Agrárias e Veterinárias, Universidade Estadual Paulista, Jaboticabal, 2001.

SANTOS, E.J.; PRADO, A.K.S.; PIZZOLATO, A.C.; MEDINA, C.L. Efeito de bioestimulantes vegetais sobre o florescimento da laranjeira pêra induzida por deficiência hídrica. In: CONGRESSO BRASILEIRO DE FISIOLOGIA VEGETAL, 9., 2003, Atibaia. Resumos... Atibaia: IAC, UNICAMP, USP, 2003. p. 226.
SILVA, J. A. B.; LAGÔA, A. M. M. A.; MACHADO, E. C. Teores de ácido abscísico (ABA) e ácido 3-indol-acético (AIA) em órgãos florais de laranjeiras com clorose variegada dos citros (CVC). CONGRESSO BRASILEIRO DE FISIOLOGIA VEGETAL, 9., 2003, Atibaia. Resumos... Atibaia: IAC, UNICAMP, USP, 2003, p. 240.

TAIZ, L.; ZEIGER, E. Fisiologia vegetal. Porto Alegre: Artmed, 2004. $135 \mathrm{p}$.

VASCONCELLOS, M.A.S.; DUARTE FILHO, J. Ecofisiologia do maracujazeiro. In: A cultura do maracujazeiro. Informe Agropecuário, Belo Horizonte, v. 21, n. 206, p. 25-28, 2000.

WATSON, D.P.; BOWERS, F.A.I. Long days produce flowers on passion fruit. Farm Science, Honolulu, v. 14, n. 2, p. 3-5, 1965. 